
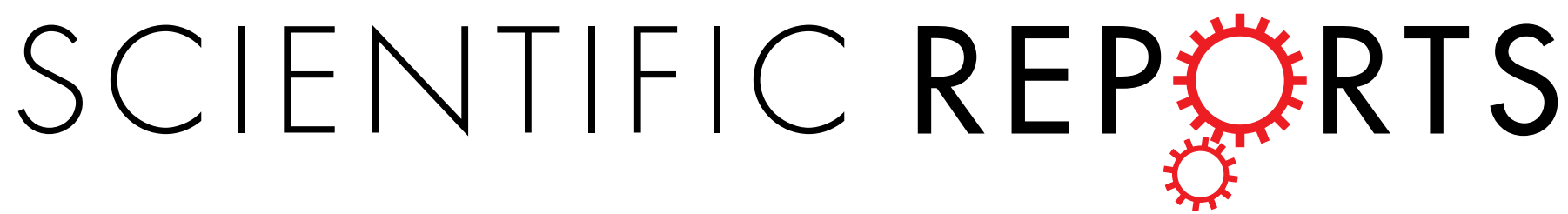

\title{
OPEN Altered serum acylcarnitine profile is associated with the status of nonalcoholic fatty liver disease \\ (NAFLD) and NAFLD-related hepatocellular carcinoma
}

Received: 7 May 2019

Accepted: 12 July 2019

Published online: 23 July 2019

\begin{abstract}
Kenichiro Enooku ${ }^{1}$, Hayato Nakagawa ${ }^{1}$, Naoto Fujiwara ${ }^{1,2}{ }^{1,2}$ Mayuko Kondo ${ }^{1}$, Tatsuya Minami ${ }^{1}$, Yujin Hoshida ${ }^{2}{ }^{2}$, Junji Shibahara ${ }^{3}$, Ryosuke Tateishi ${ }^{1}{ }^{\text {\& Kazuhiko Koike }}{ }^{1}$

Metabolic disturbance of lipids is a hallmark of nonalcoholic fatty liver disease (NAFLD). In this study, we measured the serum levels of 15 acylcarnitine species of various carbon chain lengths from 2 to 18 in 241 patients with biopsy-proven NAFLD, including 23 patients with hepatocellular carcinoma (HCC), and analyzed the relationship between serum acylcarnitine profile and NAFLD status. Longchain acylcarnitines AC14:1 and AC18:1 increased gradually with the progression of fibrosis and further increased in patients with $\mathrm{HCC}$, whereas the middle-chain acylcarnitine AC5:0 exhibited the opposite trend. In particular, AC18:1, which we previously showed to possess a tumor promoting effect, was significantly elevated in patients with HCC compared to those without HCC. In addition, long-chain acylcarntines including AC18:1 were positively correlated with serum levels of inflammatory cytokines. Although none of the acylcarnitine species were independently associated with the presence of HCC, (AC16:0 + AC18:1)/AC2:0, an index for the diagnosis of carnitine palmitoyltransferase 2 (CPT2) deficiency, was independently associated with the presence of HCC after adjusting for age and liver fibrosis stage, likely reflecting the downregulation of CPT2 in HCC tissues. Thus, serum acylcarnitine profiles changed significantly according to the status of NAFLD, which may be implicated in the pathogenesis of NAFLD.
\end{abstract}

Nonalcoholic fatty liver disease (NAFLD) is the most common chronic liver disease, affecting $25 \%$ of the general population worldwide ${ }^{1,2}$. Nonalcoholic steatohepatitis (NASH) is considered the progressive form of NAFLD and is characterized by liver steatosis, inflammation, hepatocellular injury, and fibrosis that eventually results in cirrhosis and/or hepatocellular carcinoma (HCC $)^{3}$. NASH is currently the second leading cause of liver transplantation in the USA and is estimated to become the leading indication for liver transplantation in the near future ${ }^{4}$.

NAFLD is characterized by the accumulation of various lipid species within hepatocytes. Hepatic de novo lipogenesis is paradoxically enhanced despite increased influx of lipids to the liver from insulin-resistant adipose tissue and dietary fat, which results in a marked accumulation of lipids in hepatocytes ${ }^{5,6}$. In addition, hepatic fatty acid $\beta$-oxidation (FAO) is impaired in some patients with NAFLD despite increased lipid storage ${ }^{7}$. Such disturbed lipid metabolism causes a marked accumulation of toxic lipids in hepatocytes, leading to lipotoxic cell death, inflammation, subsequent fibrosis, and $\mathrm{HCC}^{8,9}$.

We recently identified acylcarnitine species as metabolites that accumulate specifically in obesity- and $\mathrm{NASH}$-related HCC tissues using untargeted metabolomics profiling in mouse HCC model ${ }^{10}$. Acylcarnitine is the intermediate metabolite produced during FAO. Fatty acids are converted to acyl-coenzyme A (acyl-CoA) and then to acylcarnitine via conjugation to carnitine by carnitine palmitoyltransferase (CPT) 1A. After

\footnotetext{
${ }^{1}$ Department of Gastroenterology, The University of Tokyo, 7-3-1 Hongo, Bunkyo-ku, Tokyo, 113-8655, Japan. ${ }^{2}$ Liver Tumor Translational Research Program, Simmons Comprehensive Cancer Center, Division of Digestive and Liver Diseases, Department of Internal Medicine, University of Texas Southwestern Medical Center, Dallas, TX, 75390, USA. ${ }^{3}$ Department of Pathology, The University of Tokyo, 7-3-1 Hongo, Bunkyo-ku, Tokyo, 113-8655, Japan. Correspondence and requests for materials should be addressed to H.N. (email: hanakagawa-tky@umin.ac.jp)
} 
mitochondrial translocation, CPT2 converts acylcarnitine to free carnitine and acyl-CoA, which subsequently enters the FAO pathway. The accumulation of acylcarnitine in obesity- and NASH-related HCC tissues has been attributed to downregulation of CPT2, which is also observed in human NAFLD-related HCC tissues. In addition, patients with NAFLD and HCC had increased serum levels of acylcarnitine. Interestingly, serum levels of acylcarnitine increased gradually with the progression of fibrosis in patients with NAFLD and further increased in patients with NAFLD and HCC, whereas no such relationship was observed for free carnitine. Notably, several recent studies have indicated the possible effects of acylcarnitine species on disparate aspects of pathophysiology, such as inflammation and insulin resistance ${ }^{11-13}$. We also reported that oleoylcarnitine (AC18:1), one of the long-chain acylcarnitine species, functions as an oncometabolite by conferring stem cell properties to HCC cells through activation of signal transducer and activator of transcription $3^{10}$. Therefore, altered composition of acylcarnitine species may contribute to the pathophysiology of NAFLD. However, little is known about the relationships between serum acylcarnitine profiles and the status of NAFLD.

In this study, we measured serum levels of 15 acylcarnitine species of various carbon chain lengths from $\mathrm{AC} 2$ to AC18, including unsaturated acylcarnitines, using liquid chromatography-tandem mass spectrometry (LC-MS/MS) in a large cohort of 241 biopsy-proven NAFLD patients with and without HCC, and analyzed the relationships between their serum acylcarnitine profiles and NAFLD status.

\section{Results}

Patient characteristics. A total of 241 biopsy-proven NAFLD patients, including 23 patients with HCC, were enrolled in the study. Their demographic characteristics are listed in Table 1. Free carnitine (C0), acetylcarnitine (AC2:0), propionylcarnitine (AC3:0), butyrylcarnitine (AC4:0), isovalerylcarnitine (AC5:0), hydroxyisovalerylcarnitine (AC5-OH), glutarylcarnitine (AC5-DC), hexanoylcarnitine (AC6:0), octanoylcarnitine (AC8:0), decanoylcarnitine (AC10:0), dodecanoylcarnitine (AC12:0), myristoylcarnitine (AC14:0), tetradecanoylcarnitine (AC14:1), palmitoylcarnitine (AC16:0), octadecanoylcarnitine (AC18:0), and oleoylcarnitine (AC18:1) were measured by LC-MS/MS. As AC5-OH, AC5-DC, and AC14:0 were undetectable in the majority of patients with NAFLD in this cohort $(96.7 \%, 91.3 \%$, and $98.3 \%$, respectively), the values of these acylcarnitines were omitted from Table 1. Serum levels of free carnitine were similar between patients with and without HCC, whereas serum levels of total acylcarnitine were significantly higher in patients with HCC than in those without HCC. Among acylcarnitine species, only AC18:1 was significantly higher in patients with HCC than in those without HCC. In contrast, AC5:0 was significantly lower in patients with HCC than in those without HCC.

Serum levels of acylcarnitine species in patients with NAFLD. We previously reported that serum levels of total acylcarnitine increased according to the progression of fibrosis, and further increased after HCC development in patients with $\mathrm{NAFLD}^{10}$; therefore, we analyzed the associations between serum levels of acylcarnitine species and liver fibrosis stage and HCC in this cohort (Fig. 1). Consistent with our previous study, serum levels of total acylcarnitine increased gradually with the progression of fibrosis and further increased in patients with HCC in this cohort. Among acylcarnitine species, AC14:1 and AC18:1 also revealed increasing trends. In contrast, serum levels of AC5:0 decreased gradually with the progression of fibrosis and further decreased in patients with HCC. Based on these findings, we created a signature for predicting advanced stages of NAFLD using the nearest template prediction method, and the proportion of patients who had a signature of AC5:0 $0^{\text {low }}$ AC14: $1^{\text {high }} / \mathrm{AC} 18: 1^{\text {high }}$ increased significantly with NAFLD disease progression $(p=0.001$ by Cochran-Armitage test) (Fig. 2).

We also analyzed the relationships between serum levels of acylcarnitine species and liver steatosis, inflammation, and hepatocyte ballooning, but no significant correlations were detected (Supplementary Figs 1-3). Only AC5:0 was positively correlated with liver steatosis and negatively correlated with hepatocyte ballooning, both of which were of borderline significance ( $p=0.056$ and 0.088 , respectively).

Since the kidney also plays an important role in carnitine homeostasis through filtration and tubular reabsorption of carnitine and acylcarnitines ${ }^{14,15}$, we analyzed the relationships between serum levels of acylcarnitine species and estimated glomerular filtration rate (eGFR) in this cohort. Most of short- and medium-chain acylcarnitines (AC2:0, AC3:0, AC4:0, AC5:0, AC8:0, and AC10:0) in addition to C0 and total acylcarnitine were significantly negatively correlated with eGFR, while no significant correlations were detected between long-chain acylcarnitines and eGFR (Supplementary Table 1).

Relationships between serum levels of acylcarnitine species and inflammatory mediators. Acylcarnitines, particularly medium- and long-chain acylcarnitines, have been reported to activate proinflammatory signaling pathways in monocytes and induce the secretion of inflammatory cytokines and chemokines, including tumor necrosis factor $\alpha$ (TNF- $\alpha$ ), interleukin-6 (IL-6), and monocyte chemoattractant protein 1 (MCP-1) $)^{11,16,17}$, all of which have been suggested to be involved in the pathogenesis and progression of $\mathrm{NASH}$. Therefore, we evaluated the relationships between serum levels of acylcarnitine species and these inflammatory mediators in 199 NAFLD patients from this cohort (Table 2). Long-chain acylcarnitines, AC16:0, AC18:0, and AC18:1, were significantly correlated with serum TNF- $\alpha$ levels. Medium-chain acylcarnitines (AC6:0, AC8:0, AC10:0, and AC12:0) and long-chain acylcarnitines (AC14:1 and AC18:1) were positively correlated with IL-6, whereas AC5:0 was negatively correlated with IL-6. None of acylcarnitine species were significantly correlated with serum levels of MCP-1, although AC14:1 and AC16:0 had weak correlations with borderline significance.

(AC16:0 + AC18:1)/AC2:0 as a diagnostic index of CPT2 deficiency is associated with HCC in NAFLD patients. Next, we explored aylcarnitine species that were independently associated with the presence of HCC in NAFLD patients. Although serum levels of AC18:1 and AC5:0 were significantly altered in NAFLD patients with HCC (Table 1), the progression of liver fibrosis also affected the serum levels of AC18:1 and 


\begin{tabular}{|c|c|c|c|c|}
\hline Variable & Over all $(\mathrm{n}=\mathbf{2 4 1})$ & HCC $(n=23)$ & Non-HCC $(n=218)$ & $p$ value \\
\hline Gender, male, n (\%) & $135(56)$ & $14(61)$ & $121(56)$ & 0.66 \\
\hline Age (year) & $58(45-67)$ & $72(65-76)$ & $55(44-65)$ & $<0.001$ \\
\hline BMI $\left(\mathrm{kg} / \mathrm{m}^{2}\right)$ & $28.3(25.5-31.2)$ & $28.0(25.4-29.6)$ & $28.3(25.6-31.2)$ & 0.73 \\
\hline Platelet count $\left(\times 10^{3} / \mu \mathrm{L}\right)$ & $211(166-244)$ & $126(83-182)$ & $215(173-250)$ & $<0.001$ \\
\hline Albumin $(\mathrm{g} / \mathrm{dL})$ & $4.0(3.7-4.2)$ & $3.4(3.2-4.0)$ & $4.0(3.8-4.2)$ & $<0.001$ \\
\hline AST (IU/L) & $39(28-60)$ & $35(27-39)$ & $40(28-62)$ & 0.14 \\
\hline ALT (IU/L) & $53(33-86)$ & $25(19-33)$ & $57(37-91)$ & $<0.001$ \\
\hline Fibrosis $(0 / 1 / 2 / 3 / 4), \mathrm{n}(\%)$ & $38 / 74 / 41 / 63 / 25(16 / 31 / 17 / 26 / 10)$ & $1 / 0 / 2 / 10 / 10(4 / 0 / 9 / 43 / 43)$ & $37 / 74 / 39 / 53 / 15(17 / 34 / 18 / 24 / 7)$ & $<0.001$ \\
\hline Steatosis $(1 / 2 / 3), \mathrm{n}(\%)$ & $133 / 73 / 35(55 / 30 / 15)$ & $21 / 1 / 2(91 / 4 / 4)$ & $112 / 72 / 34(51 / 33 / 16)$ & $<0.001$ \\
\hline $\begin{array}{l}\text { Lobular inflammation } \\
(0 / 1 / 2 / 3), \mathrm{n}(\%)\end{array}$ & $16 / 152 / 67 / 6(7 / 63 / 28 / 2)$ & $1 / 18 / 4 / 0(4 / 78 / 17 / 0)$ & $15 / 134 / 63 / 6(7 / 61 / 29 / 3)$ & 0.56 \\
\hline $\begin{array}{l}\text { Hepatocyte ballooning } \\
(0 / 1 / 2), \mathrm{n}(\%)\end{array}$ & $74 / 117 / 50(31 / 49 / 20)$ & $6 / 13 / 4(26 / 57 / 17)$ & $68 / 104 / 46(31 / 48 / 21)$ & 0.8 \\
\hline $\begin{array}{l}\text { Total Acylcarnitine } \\
\text { (enzymatic cycling } \\
\text { method) }(\mu \mathrm{mol} / \mathrm{L})\end{array}$ & $9.7(8.0-11.5)$ & $10.2(9.5-13.2)$ & $9.6(7.9-11.2)$ & 0.01 \\
\hline $\begin{array}{l}\text { Free carnitine (enzymatic } \\
\text { cycling method) }(\mu \mathrm{mol} / \mathrm{L})\end{array}$ & $53.7(48.7-58.3)$ & $56.5(44.7-59.0)$ & $53.4(48.7-58.2)$ & 0.69 \\
\hline \multicolumn{5}{|c|}{ Acylcarnitine species (LC-MS/MS) } \\
\hline $\mathrm{C} 0(\mu \mathrm{mol} / \mathrm{L})$ & $50.6(42.8-55.9)$ & $51(40.3-60.7)$ & $50.6(43.1-55.8)$ & 0.86 \\
\hline AC2:0 $(\mu \mathrm{mol} / \mathrm{L})$ & $7.31(5.98-9.23)$ & $7.16(6.24-8.83)$ & $7.36(5.93-9.26)$ & 0.94 \\
\hline AC3:0 $(\mu \mathrm{mol} / \mathrm{L})$ & $0.423(0.355-0.524)$ & $0.466(0.371-0.578)$ & $0.422(0.352-0.52)$ & 0.38 \\
\hline AC4:0 $(\mu \mathrm{mol} / \mathrm{L})$ & $0.153(0.124-0.188)$ & $0.149(0.113-0.178)$ & $0.153(0.126-0.188)$ & 0.42 \\
\hline AC5:0 $(\mu \mathrm{mol} / \mathrm{L})$ & $0.072(0-0.091)$ & $0.06(0-0.075)$ & $0.073(0-0.092)$ & 0.03 \\
\hline AC6:0 $(\mu \mathrm{mol} / \mathrm{L})$ & $0(0-0.05)$ & $0(0-0.063)$ & $0(0-0.056)$ & 0.45 \\
\hline AC8:0 $(\mu \mathrm{mol} / \mathrm{L})$ & $0.089(0.066-0.138)$ & $0.09(0.079-0.116)$ & $0.08(0.065-0.139)$ & 0.38 \\
\hline AC10:0 $(\mu \mathrm{mol} / \mathrm{L})$ & $0.134(0.093-0.219)$ & $0.134(0.115-0.172)$ & $0.134(0.09-0.219)$ & 0.48 \\
\hline $\mathrm{AC} 12: 0(\mu \mathrm{mol} / \mathrm{L})$ & $0(0-0.064)$ & $0(0-0.063)$ & $0(0-0.064)$ & 0.98 \\
\hline $\mathrm{AC} 14: 1(\mu \mathrm{mol} / \mathrm{L})$ & $0.062(0.085-0.116)$ & $0.097(0.081-0.131)$ & $0.085(0.015-0.11)$ & 0.08 \\
\hline $\mathrm{AC} 16: 0(\mu \mathrm{mol} / \mathrm{L})$ & $0.114(0.098-0.136)$ & $0.114(0.103-0.134)$ & $0.114(0.098-0.135)$ & 0.64 \\
\hline $\mathrm{AC} 18: 0(\mu \mathrm{mol} / \mathrm{L})$ & $0.042(0.035-0.048)$ & $0.038(0.034-0.046)$ & $0.042(0.035-0.048)$ & 0.4 \\
\hline AC18:1 $(\mu \mathrm{mol} / \mathrm{L})$ & $0.661(0.554-0.807)$ & $0.73(0.645-0.87)$ & $0.649(0.543-0.805)$ & 0.03 \\
\hline \multicolumn{5}{|l|}{ Tumor status } \\
\hline Tumor size $(\mathrm{cm})$ & & $2.1(1.8-2.8)$ & & \\
\hline $\begin{array}{l}\text { Number of nodules } \\
\text { (Solitary/2 or } 3 \text { nodules), } \\
\mathrm{n}(\%)\end{array}$ & & $16 / 7(70 / 30)$ & & \\
\hline
\end{tabular}

Table 1. Baseline characteristics of patients with NAFLD with or without HCC. Data are expressed as median $\left(25^{\text {th }}-75^{\text {th }}\right.$ percentiles) unless otherwise indicated. HCC, hepatocellular carcinoma; BMI, body mass index; AST, aspartate aminotransferase; ALT, alanine transaminase.

AC5:0 (Fig. 1), and thus serum AC18:1 and AC5:0 were not independent indicators for the presence of HCC after adjusting for liver fibrosis stage. We previously showed that the accumulation of acylcarnitine in NAFLD-related HCC tissues was caused by downregulation of CPT2, whereas the expression of CPT2 in non-tumorous tissues was not altered by liver fibrosis ${ }^{10}$. Based on these findings, we adopted the index (AC16:0 + AC18:1)/AC2:0 to diagnose congenital deficiency of CPT $2^{18}$ as an indicator for the presence of HCC in NAFLD patients. As shown in Fig. 3a,b, although the (AC16:0 + AC18:1)/AC2:0 index was not affected by the degree of liver fibrosis, the index was significantly higher in NAFLD patients with HCC compared to those without HCC. Furthermore, the (AC16:0 + AC18:1)/AC2:0 index was an independent factor associated with the presence of HCC after adjusting for age and liver fibrosis stage (Table 3 ).

\section{Discussion}

In the present study, serum acylcarnitine profiles were associated with various aspects of the pathophysiology of NAFLD. Especially, AC18:1 was significantly positively correlated with serum levels of inflammatory cytokines and significantly increased in NAFLD patients with HCC, which were consistent with our previous mouse experiments ${ }^{10}$. To date, several studies have reported associations between acylcarnitine profiles and liver disease $\mathrm{e}^{19-22}$. However, those studies included relatively small numbers of NAFLD patients, and, to the best of our knowledge, this is the largest study to assess the relationships between acylcarnitine species and the status of NAFLD.

Serum levels of unsaturated long-chain acylcarnitine species, such as AC14:1 and AC18:1 increased with the progression of liver fibrosis and HCC, whereas medium-chain acylcarnitine AC5:0 showed the opposite trend in 
Total acylcarnitine
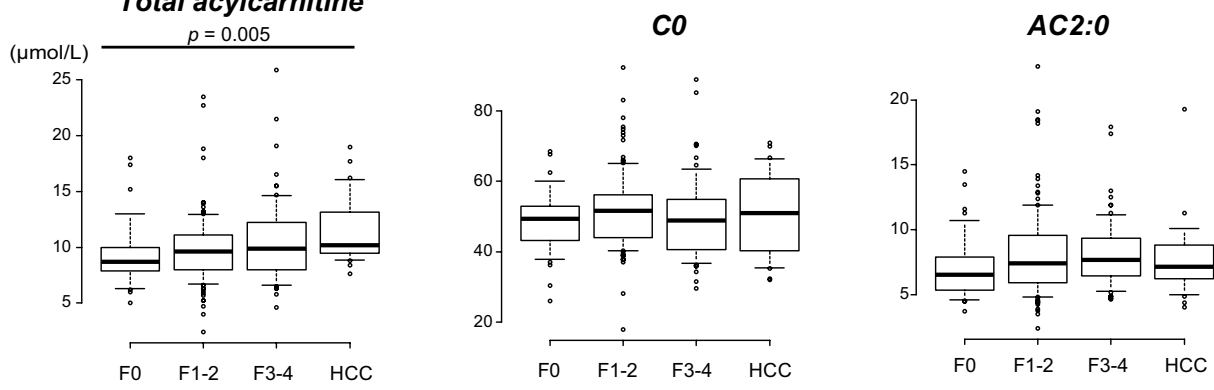

AC3:0
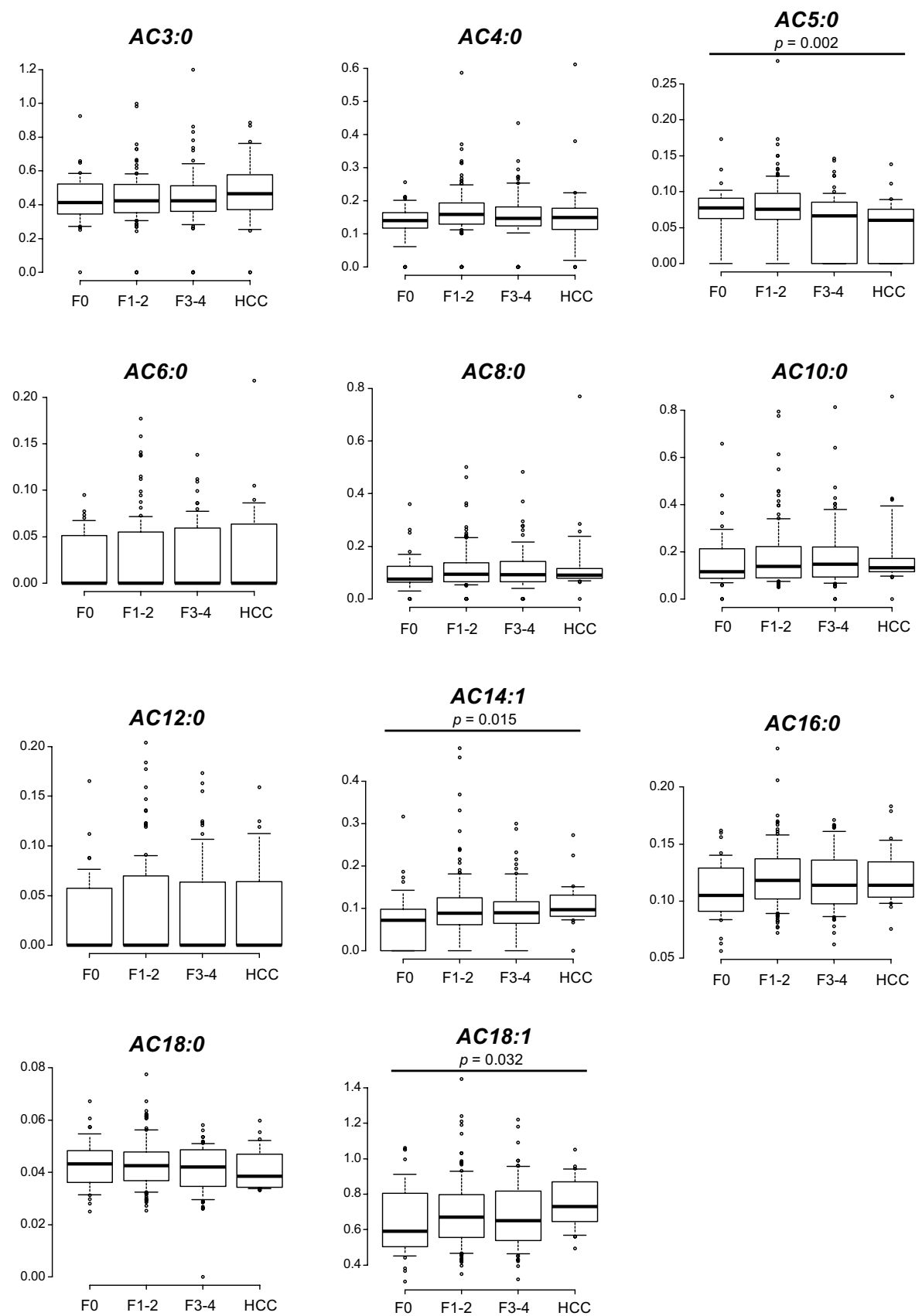

Figure 1. Serum levels of acylcarnitine species in nonalcoholic fatty liver disease (NAFLD) patients with and without hepatocellular carcinoma (HCC). Patients without HCC were classified according to their fibrosis stage. The box plot indicates the mean (horizontal line), interquartile range (box), $10^{\text {th }}$ and $90^{\text {th }}$ percentiles (whiskers), and outliers outside the $10^{\text {th }}$ and $90^{\text {th }}$ percentiles (dots). 


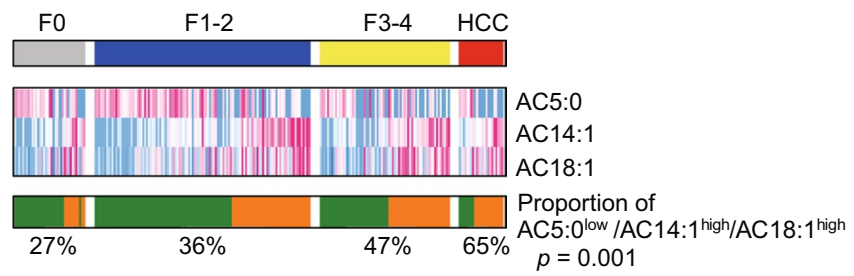

Figure 2. The proportion of patients with the AC5:0 $0^{\text {low }} / A C 14: 1^{\text {high }} / \mathrm{AC} 18: 1^{\text {high }}$ signature according to NAFLD status.

a

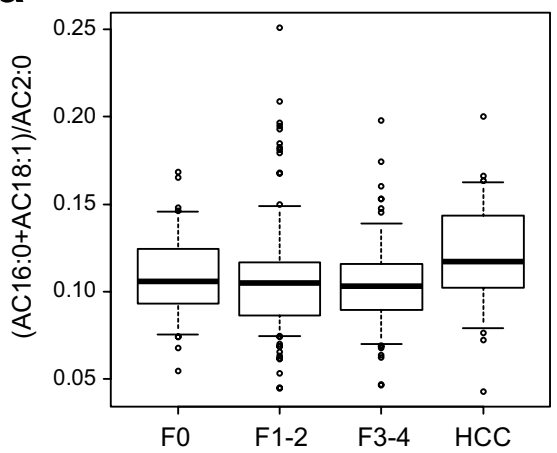

b

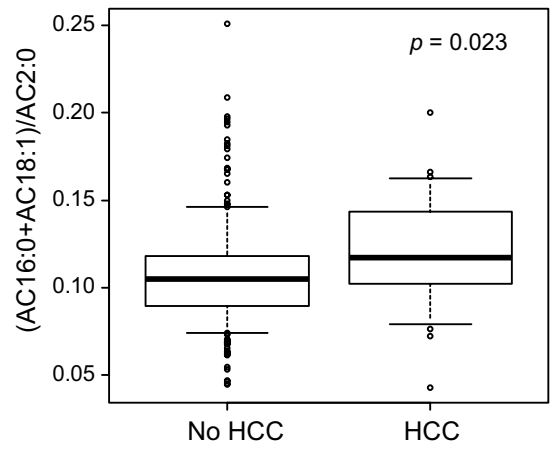

Figure 3. The values of the (AC16:0 + AC18:1)/AC2:0 index in NAFLD patients with or without HCC. Patients without HCC were classified according to their fibrosis stage (a) and non-classified (b). The box plot indicates the mean (horizontal line), interquartile range (box), $10^{\text {th }}$ and $90^{\text {th }}$ percentiles (whiskers), and outliers outside the $10^{\text {th }}$ and $90^{\text {th }}$ percentiles (dots).

\begin{tabular}{|c|c|c|c|c|c|c|}
\hline \multirow[b]{2}{*}{$\begin{array}{l}\text { Acylcarnitine } \\
\text { species }\end{array}$} & \multicolumn{2}{|l|}{ TNFo } & \multicolumn{2}{|l|}{ IL-6 } & \multicolumn{2}{|l|}{ MCP1 } \\
\hline & $\begin{array}{l}\text { Spearman's } \\
\text { Rho }\end{array}$ & $p$ value & $\begin{array}{l}\text { Spearman's } \\
\text { Rho }\end{array}$ & $p$ value & $\begin{array}{l}\text { Spearman's } \\
\text { Rho }\end{array}$ & $p$ value \\
\hline $\mathrm{CO}$ & 0.05 & 0.48 & 0.03 & 0.68 & 0.04 & 0.61 \\
\hline AC2:0 & 0.07 & 0.31 & 0.10 & 0.15 & 0.05 & 0.49 \\
\hline AC3:0 & 0.04 & 0.55 & 0.03 & 0.71 & 0.09 & 0.22 \\
\hline AC4:0 & 0.11 & 0.14 & -0.01 & 0.91 & 0.10 & 0.18 \\
\hline AC5:0 & 0.04 & 0.56 & -0.16 & 0.02 & 0.00 & 0.95 \\
\hline AC6:0 & 0.02 & 0.78 & 0.14 & 0.04 & 0.07 & 0.34 \\
\hline AC8:0 & -0.01 & 0.90 & 0.19 & 0.01 & 0.06 & 0.41 \\
\hline AC10:0 & -0.02 & 0.82 & 0.21 & 0.003 & 0.05 & 0.48 \\
\hline AC12:0 & -0.05 & 0.47 & 0.19 & 0.01 & 0.09 & 0.20 \\
\hline AC14:1 & 0.05 & 0.46 & 0.27 & $<0.001$ & 0.13 & 0.08 \\
\hline AC16:0 & 0.25 & $<0.001$ & 0.13 & 0.06 & 0.12 & 0.09 \\
\hline AC18:0 & 0.17 & 0.02 & -0.03 & 0.68 & 0.06 & 0.43 \\
\hline AC18:1 & 0.24 & $<0.001$ & 0.20 & 0.004 & 0.11 & 0.12 \\
\hline
\end{tabular}

Table 2. Correlations between acylcarnitine species and inflammatory mediators.

our cohort. As a result, the proportion of patients who had the AC5:0 $0^{\text {low }} / \mathrm{AC} 14: 1^{\text {high }} / \mathrm{AC} 18: 1^{\text {high }}$ signature increased significantly with the progression of NAFLD. In contrast, serum acylcarnitine profiles did not affect liver steatosis, inflammation, or hepatocyte ballooning. Pérez-Carreras et al. reported an increase in long-chain acylcarnitine and a decrease in short-chain acylcarnitine in the serum of patients with NASH compared to control subjects, and Peng et al. reported that only AC18:1 increased in the serum of patients with NASH compared to obese control subjects ${ }^{7,20}$. These findings are largely consistent with our results. However, as these studies contained small numbers of patients with NASH (43 and 32 patients, respectively), no detailed analysis of the relationship between the pathological findings of NAFLD and the serum acylcarnitine profiles was performed. Increased long-chain acylcarnitine and decreased short- and middle-chain acylcarnitine were observed in patients with chronic hepatitis B virus (HBV) infection ${ }^{19}$, consistent with our results. Therefore, such changes in serum acylcarnitine profiles may be commonly caused by the progression of liver fibrosis, although further studies are needed. 


\begin{tabular}{|l|l|l|l|l|}
\hline \multirow{2}{*}{ Variable } & \multicolumn{3}{l|}{ Univariate analyses } & \multicolumn{2}{l|}{ Multivariate analyses } \\
\cline { 2 - 6 } & Odds ratio & $\boldsymbol{p}$ value & Odds ratio & $\boldsymbol{p}$ value \\
\hline Age $($ per 1 year) & $1.17(1.09-1.25)$ & $<0.001$ & $1.16(1.07-1.25)$ & $<0.001$ \\
\hline Gender, male & $1.25(0.51-3.00)$ & 0.62 & & \\
\hline BMI $\left(\right.$ per $\left.1 \mathrm{~kg} / \mathrm{m}^{2}\right)$ & $0.99(0.9-1.09)$ & 0.91 & & \\
\hline Fibrosis stage $($ per 1 stage) & $3.48(2.05-5.91)$ & $<0.001$ & $2.87(1.50-5.51)$ & 0.002 \\
\hline AC5:0 $($ per $0.01 \mu \mathrm{mol} / \mathrm{L})$ & $0.89(0.81-1.00)$ & 0.03 & & \\
\hline AC18:1 $($ per $0.1 \mu \mathrm{mol} / \mathrm{L})$ & $1.19(0.96-1.47)$ & 0.1 & & \\
\hline (AC16:0 + AC18:1)/AC2:0 $($ per 0.01$)$ & $1.12(1.00-1.27)$ & 0.05 & $1.25(1.05-1.48)$ & 0.01 \\
\hline
\end{tabular}

Table 3. Factors associated with HCC in patients with NAFLD.

We also found that (AC16:0 + AC18:1)/AC2:0, a diagnostic index for congenital deficiency of CPT2, was independently associated with HCC in patients with NAFLD. Because AC2:0 is an end product of mitochondrial $\mathrm{FAO}$, a high (AC16:0 + AC18:1)/AC2:0 index reflects increased generation and decreased degradation of acylcarnitine. In patients with NAFLD, a large amount of fatty acids is delivered to the liver as materials of acylcarnitine, whereas FAO is frequently suppressed in HCC tissue due to downregulation of CPT2, which may result in a higher index value. Consistent with our results, Lu et al. recently reported that the serum and tissue levels of long-chain acylcarnitine species increased but those of short- and middle-chain acylcarnitine species decreased in patients with hepatitis virus-related HCC, and such changes in acylcarnitine profiles were caused by downregulation of $\mathrm{CPT}^{22}$. In contrast, Zhou et al. reported that serum acylcarnitine profiles were similar between patients with advanced fibrosis and HCC in those with chronic HBV infection ${ }^{19}$. Such differences among studies may be attributed to the degree of CPT2 downregulation in HCC. As we previously reported ${ }^{10}$, downregulation of CPT2 is more frequently seen in steatohepatitic HCC that often occurs in patients with steatohepatitis with or without hepatitis virus infection ${ }^{23,24}$. Peroxisome proliferator-activated receptor $\alpha(P P A R \alpha)$ mainly regulates the expression of CPT2, and a recent study showed that activation of $\beta$-catenin plays a critical role in the upregulation of PPAR $\alpha$ and CPT2 in $\mathrm{HCC}^{25}$. Importantly, the frequency of mutation and activation of $\beta$-catenin is significantly low in steatohepatitic $\mathrm{HCC}^{26,27}$. Therefore, the (AC16:0 + AC18:1)/AC2:0 index may be more useful for predicting HCC in patients with steatohepatitis and could be a surrogate marker for activation states of $\beta$-catenin and FAO in HCC. Furthermore, AC18:1, which we previously showed possesses a tumor promoting effect in mouse models ${ }^{10}$, increased significantly in NAFLD patients with HCC, suggesting that AC18:1 may act as an oncometabolite in human hepatocarcinogenesis.

Various acylcarnitine species were associated with serum levels of inflammatory cytokines in patients with NAFLD. TNF- $\alpha$ and IL-6 play pivotal roles in NAFLD progression and HCC development in mouse models ${ }^{28-31}$. Acylcarnitines, particularly medium- and long-chain acylcarnitines, reportedly induce the secretion of inflammatory cytokines through activation of the Toll-like receptor/MyD88 signaling-mediated nuclear factor $\kappa \mathrm{B}$ pathway ${ }^{11,16,17}$. Therefore, acylcarnitine released from hepatocytes may also be involved in the pathogenesis of NAFLD by stimulating the secretion of inflammatory cytokines from immune cells.

We also analyzed the relationships between serum levels of acylcarnitine species and renal function in NAFLD patients and found that short- and medium-chain acylcarnitines, but not long-chain acylcarnitines, were significantly negatively correlated with eGFR. Although the mechanism is unclear, these findings were consistent with a previous study analyzing the patients with diabetic kidney disease ${ }^{32}$. In other words, long-chain acylcarnitines can reflect the status of NAFLD without being affected by renal function.

This study had several limitations. First, data on the amounts of acylcarnitine species in the liver and HCC tissues were not available. As acylcarnitine species can also be released from other tissues, the changes in serum acylcarnitine profiles may not directly reflect the status of acylcarnitine metabolism and FAO in the liver and HCC tissues. In particular, skeletal muscle is another major source of acylcarnitines, but we did not have data on skeletal muscle volume in our NAFLD cohort. Since sarcopenia is associated with poor outcomes in patients with liver diseases ${ }^{33}$, the relationships between serum acylcarnitine profiles and the skeletal muscle volume need to be explored. Second, although carnitine homeostasis is maintained by dietary intake (mainly meat products) as well as endogenous synthesis and renal reabsorption ${ }^{34}$, we did not have data on dietary habits in this cohort. Third, although we and another group previously identified downregulation of CPT2 as a critical factor for acylcarnitine accumulation in HCC tissues ${ }^{10,22}$, the mechanism underlying the changes in serum acylcarnitine profiles during the progression of NAFLD remains unclear. One possibility is that FAO in patients with advanced-stage NASH may be impaired in the process of acyl-CoA dehydrogenation that is catalyzed by acyl-CoA dehydrogenases including very long-chain acyl-CoA dehydrogenase (VLCAD), long-chain acyl-CoA dehydrogenase (LCAD), and medium-chain acyl-CoA dehydrogenase (MCAD). In fact, patients with VLCAD deficiency show significant elevations of serum long-chain acylcarnitine species ${ }^{35}$. Since the expressions and functions of acyl-CoA dehydrogenases in patients with NAFLD are poorly understood, further studies are needed.

In summary, serum acylcarnitine profiles changed significantly with the progression of NAFLD. The roles of acylcarnitines in the pathogenesis of NAFLD merit further investigation.

\section{Methods}

Patients. Levels of acylcarnitine species were analyzed in serum samples collected from 241 patients with biopsy-proven NAFLD with $(n=23)$ and without HCC $(n=218)$ at the University of Tokyo Hospital from November 2011 to December 2017. The recruitment criteria of liver biopsy for patients without HCC were as follows: liver transient elastography measured by FibroScan $>7.0 \mathrm{kPa}$; persistent elevations of serum aspartate 
aminotransferase and alanine aminotransferase above the upper limit for at least 6 months; fatty liver diagnosis based on ultrasound examination showing an increase in hepatorenal contrast, history of alcohol consumption $<30 \mathrm{~g} /$ day for males or $<20 \mathrm{~g} /$ day for females; seronegativity for hepatitis B virus surface antigen and hepatitis C virus antibody; and absence of autoimmune hepatitis, primary biliary cholangitis, primary sclerosing cholangitis, Budd-Chiari syndrome, Wilson disease, and drug-induced liver injury. All patients with HCC were treated with radiofrequency ablation and simultaneously underwent biopsies of non-tumor liver tissues to diagnose underlying liver diseases. All serum samples were obtained in the morning on the day of the liver biopsy and/ or radiofrequency ablation after an overnight fast. Serum samples were frozen at $-70{ }^{\circ} \mathrm{C}$ until assay. Among the 241 serum samples, 180, including 18 samples obtained from patients with HCC, were the same samples as used in our previous study analyzing serum total acylcarnitine levels ${ }^{10}$, and 61 samples, including five samples obtained from patients with HCC, were newly added in this study. None of the patients included in this study were supplemented with L-carnitine. eGFR was calculated using the following formula established by the working group of the Japanese Chronic Kidney Disease Initiative: eGFR $\left(\mathrm{mL} / \mathrm{min} / 1.73 \mathrm{~m}^{2}\right)=194 \times($ serum creatinine $)^{-1.094} \times(\text { age })^{-0.287}(\times 0.739 \text { for women })^{36}$. The study protocol conformed to the ethical guidelines of the Declaration of Helsinki and was approved by the Ethics Committee of the University of Tokyo. All patients provided informed consent.

Serum concentrations of acylcarnitine species. The serum concentrations of acylcarnitines were analyzed by LC-MS/MS at Sekisui Medical Co., Ltd (Tokyo, Japan). The NeoSMAAT AC kit, a new reagent composed of 15 types of acylcarnitine species, was used to create the calibration curves to measure the serum concentrations of acylcarnitine species more accurately.

Serum concentrations of total acylcarnitine, free carnitine, and inflammatory mediators. Serum levels of total acylcarnitine and free carnitine were measured using the enzymatic cycling method at SRL Inc. (Tokyo, Japan). Serum levels of TNF- $\alpha$, IL-6, and MCP-1 were also measured at SRL Inc. Among the 241 serum samples, 42 were not subjected to analyses of inflammatory mediators due to a limited sample volume.

Histopathological assessment. Liver biopsy samples were obtained from the right lobe using a $16 \mathrm{G}$ needle with a 20-mm long biopsy specimen notch. All samples were assessed by experienced pathologists blinded to the clinical data and the study design. Liver histology was assessed according to the criteria of the Nonalcoholic Steatohepatitis Clinical Research Network ${ }^{37}$.

Statistical analyses. Intergroup differences of continuous and categorical variables were tested using the Mann-Whitney $U$-test and Fisher's exact test, respectively. The increasing and decreasing tendencies of acylcarnitine species across the categorical data were assessed using the Jonckheere-Terpstra trend test. Serum acylcarnitine signature-based prediction of advanced NAFLD was determined using the nearest template prediction algorithm ${ }^{38}$. In this analysis, patients were classified into the two groups based on the "cosine distance" from the hypothetical template. If the distance is greater than 0 , the patient was recognized as high. Correlations between serum levels of acylcarnitine species and continuous variables were analyzed using Spearman's rank correlation coefficient analysis. Univariate and multivariate analyses were performed for the presence of HCC using a logistic regression model with a stepwise procedure. Variables with $P$-values $<0.1$ were included in the multivariate analysis. A two-tailed $p<0.05$ was considered significant. All data analyses were performed using the $R$ statistical software (www.r-project.org).

\section{Data Availability}

The datasets generated during and/or analyzed during the current study are available from the corresponding author on reasonable request.

\section{References}

1. Younossi, Z. M. et al. Global epidemiology of nonalcoholic fatty liver disease-Meta-analytic assessment of prevalence, incidence, and outcomes. Hepatology (Baltimore, Md.) 64, 73-84, https://doi.org/10.1002/hep.28431 (2016).

2. Lebeaupin, C. et al. Endoplasmic reticulum stress signalling and the pathogenesis of non-alcoholic fatty liver disease. J Hepatol 69, 927-947, https://doi.org/10.1016/j.jhep.2018.06.008 (2018).

3. Nakagawa, H. Recent advances in mouse models of obesity- and nonalcoholic steatohepatitis-associated hepatocarcinogenesis. World J Hepatol 7, 2110-2118, https://doi.org/10.4254/wih.v7.i17.2110 (2015).

4. Noureddin, M. et al. NASH Leading Cause of Liver Transplant in Women: Updated Analysis of Indications For Liver Transplant and Ethnic and Gender Variances. The American journal of gastroenterology 113, 1649-1659, https://doi.org/10.1038/s41395-018-00886 (2018).

5. Donnelly, K. L. et al. Sources of fatty acids stored in liver and secreted via lipoproteins in patients with nonalcoholic fatty liver disease. The Journal of clinical investigation 115, 1343-1351, https://doi.org/10.1172/jci23621 (2005).

6. Friedman, S. L., Neuschwander-Tetri, B. A., Rinella, M. \& Sanyal, A. J. Mechanisms of NAFLD development and therapeutic strategies. Nature medicine 24, 908-922, https://doi.org/10.1038/s41591-018-0104-9 (2018).

7. Perez-Carreras, M. et al. Defective hepatic mitochondrial respiratory chain in patients with nonalcoholic steatohepatitis. Hepatology (Baltimore, Md.) 38, 999-1007, https://doi.org/10.1053/jhep.2003.50398 (2003).

8. Nakagawa, H. et al. ER stress cooperates with hypernutrition to trigger TNF-dependent spontaneous HCC development. Cancer Cell 26, 331-343, 10.1016/j.ccr.2014.07.001 (2014).

9. Marra, F. \& Svegliati-Baroni, G. Lipotoxicity and the gut-liver axis in NASH pathogenesis. J Hepatol 68, 280-295, https://doi. org/10.1016/j.jhep.2017.11.014 (2018).

10. Fujiwara, N. et al. CPT2 downregulation adapts HCC to lipid-rich environment and promotes carcinogenesis via acylcarnitine accumulation in obesity. Gut 67, 1493-1504, https://doi.org/10.1136/gutjnl-2017-315193 (2018).

11. Rutkowsky, J. M. et al. Acylcarnitines activate proinflammatory signaling pathways. American journal of physiology. Endocrinology and metabolism 306, E1378-1387, https://doi.org/10.1152/ajpendo.00656.2013 (2014) 
12. Aguer, C. et al. Acylcarnitines: potential implications for skeletal muscle insulin resistance. FASEB journal: official publication of the Federation of American Societies for Experimental Biology 29, 336-345, https://doi.org/10.1096/f.14-255901 (2015).

13. McCoin, C. S., Knotts, T. A. \& Adams, S. H. Acylcarnitines-old actors auditioning for new roles in metabolic physiology. Nat Rev Endocrinol 11, 617-625, https://doi.org/10.1038/nrendo.2015.129 (2015).

14. Fouque, D. et al. Relationship between serum carnitine, acylcarnitines, and renal function in patients with chronic renal disease. Journal of renal nutrition: the official journal of the Council on Renal Nutrition of the National Kidney Foundation 16, 125-131, https://doi.org/10.1053/j.jrn.2006.01.004 (2006).

15. Matera, M. et al. History of L-carnitine: implications for renal disease. Journal of renal nutrition: the official journal of the Council on Renal Nutrition of the National Kidney Foundation 13, 2-14 (2003).

16. Sampey, B. P. et al. Metabolomic profiling reveals mitochondrial-derived lipid biomarkers that drive obesity-associated inflammation. PloS one 7, e38812, https://doi.org/10.1371/journal.pone.0038812 (2012).

17. Adams, S. H. et al. Plasma acylcarnitine profiles suggest incomplete long-chain fatty acid beta-oxidation and altered tricarboxylic acid cycle activity in type 2 diabetic African-American women. The Journal of nutrition 139, 1073-1081, https://doi.org/10.3945/ in.108.103754 (2009).

18. Gempel, K. et al. Screening for carnitine palmitoyltransferase II deficiency by tandem mass spectrometry. Journal of inherited metabolic disease 25, 17-27 (2002).

19. Zhou, L. et al. Serum metabolomics reveals the deregulation of fatty acids metabolism in hepatocellular carcinoma and chronic liver diseases. Analytical and bioanalytical chemistry 403, 203-213, https://doi.org/10.1007/s00216-012-5782-4 (2012).

20. Peng, K. Y. et al. Mitochondrial dysfunction-related lipid changes occur in nonalcoholic fatty liver disease progression. Journal of lipid research 59, 1977-1986, https://doi.org/10.1194/jlr.M085613 (2018).

21. Romero-Ibarguengoitia, M. E. et al. Family history and obesity in youth, their effect on acylcarnitine/aminoacids metabolomics and non-alcoholic fatty liver disease (NAFLD). Structural equation modeling approach. PloS one 13, e0193138, https://doi.org/10.1371/ journal.pone.0193138 (2018).

22. Lu, X. et al. Metabolic profiling analysis upon acylcarnitines in tissues of hepatocellular carcinoma revealed the inhibited carnitine shuttle system caused by the downregulated carnitine palmitoyltransferase 2. Molecular carcinogenesis, https://doi.org/10.1002/ mc.22967 (2019).

23. Salomao, M. et al. The steatohepatitic variant of hepatocellular carcinoma and its association with underlying steatohepatitis. Hum Pathol 43, 737-746, https://doi.org/10.1016/j.humpath.2011.07.005 (2012).

24. Shibahara, J., Ando, S., Sakamoto, Y., Kokudo, N. \& Fukayama, M. Hepatocellular carcinoma with steatohepatitic features: a clinicopathological study of Japanese patients. Histopathology 64, 951-962, https://doi.org/10.1111/his.12343 (2014).

25. Senni, N. et al. beta-catenin-activated hepatocellular carcinomas are addicted to fatty acids. Gut. https://doi.org/10.1136/ gutjnl-2017-315448 (2018).

26. Ando, S., Shibahara, J., Hayashi, A. \& Fukayama, M. beta-catenin alteration is rare in hepatocellular carcinoma with steatohepatitic features: immunohistochemical and mutational study. Virchows Archiv: an international journal of pathology 467, 535-542, https:// doi.org/10.1007/s00428-015-1836-2 (2015).

27. Nakagawa, H. et al. Lipid Metabolic Reprogramming in Hepatocellular Carcinoma. Cancers 10, https://doi.org/10.3390/ cancers10110447 (2018).

28. Mas, E. et al. IL-6 deficiency attenuates murine diet-induced non-alcoholic steatohepatitis. PloS one 4, e7929, https://doi. org/10.1371/journal.pone.0007929 (2009).

29. Park, E. J. et al. Dietary and genetic obesity promote liver inflammation and tumorigenesis by enhancing IL-6 and TNF expression. Cell 140, 197-208, https://doi.org/10.1016/j.cell.2009.12.052 (2010).

30. He, G. et al. Identification of liver cancer progenitors whose malignant progression depends on autocrine IL-6 signaling. Cell 155, 384-396, https://doi.org/10.1016/j.cell.2013.09.031 (2013).

31. Nakagawa, H. \& Maeda, S. Inflammation- and stress-related signaling pathways in hepatocarcinogenesis. World J Gastroenterol 18, 4071-4081, https://doi.org/10.3748/wjg.v18.i31.4071 (2012).

32. Liu, J. J. et al. Profiling of Plasma Metabolites Suggests Altered Mitochondrial Fuel Usage and Remodeling of Sphingolipid Metabolism in Individuals With Type 2 Diabetes and Kidney Disease. Kidney international reports 2, 470-480, https://doi. org/10.1016/j.ekir.2016.12.003 (2017).

33. Fujiwara, N. et al. Sarcopenia, intramuscular fat deposition, and visceral adiposity independently predict the outcomes of hepatocellular carcinoma. J Hepatol 63, 131-140, https://doi.org/10.1016/j.jhep.2015.02.031 (2015).

34. Rebouche, C. J. Kinetics, pharmacokinetics, and regulation of L-carnitine and acetyl-L-carnitine metabolism. Annals of the New York Academy of Sciences 1033, 30-41, https://doi.org/10.1196/annals.1320.003 (2004).

35. Wood, J. C. et al. Diagnosis of very long chain acyl-dehydrogenase deficiency from an infant's newborn screening card. Pediatrics 108, E19, https://doi.org/10.1542/peds.108.1.e19 (2001).

36. Matsuo, S. et al. Revised equations for estimated GFR from serum creatinine in Japan. American journal of kidney diseases: the official journal of the National Kidney Foundation 53, 982-992, https://doi.org/10.1053/j.ajkd.2008.12.034 (2009).

37. Kleiner, D. E. et al. Design and validation of a histological scoring system for nonalcoholic fatty liver disease. Hepatology (Baltimore, Md.) 41, 1313-1321, https://doi.org/10.1002/hep.20701 (2005).

38. Hoshida, Y. Nearest template prediction: a single-sample-based flexible class prediction with confidence assessment. PloS one 5, e15543, https://doi.org/10.1371/journal.pone.0015543 (2010).

\section{Acknowledgements}

This study was supported by JSPS KAKENHI Grant number 18H02789, Uehara Memorial Foundation, Daiichi Sankyo Foundation of Life Science, Bristol-Myers Squibb Research Grant, Takeda Science Foundation, Ichiro Kanehara Foundation, SGH Foundation, MSD Life Science Foundation, The Naito Foundation, Kobayashi Foundation for Cancer Research (H.N.), Program for Basic and Clinical Research on Hepatitis from Japan Agency for Medical Research and Development, AMED under Grant Number JP19fk0210059 (H.N.) JP18fk0210040 (H.N. and K.K.), and AMED-CREST under Grant Number JP17gm0710004 (H.N.).

\section{Author Contributions}

K.E. and H.N. designed study; K.E., M.K. and T.M. collected samples; K.E., H.N. and F.N. analyzed data; J.S. analyzed pathological findings; K.E. and H.N. wrote the paper; Y.H. and R.T. contributed to critical revision of the manuscript for important intellectual content; K.K. supervised the entire project.

\section{Additional Information}

Supplementary information accompanies this paper at https://doi.org/10.1038/s41598-019-47216-2.

Competing Interests: The authors declare no competing interests. 
Publisher's note: Springer Nature remains neutral with regard to jurisdictional claims in published maps and institutional affiliations.

(c) (i) Open Access This article is licensed under a Creative Commons Attribution 4.0 International License, which permits use, sharing, adaptation, distribution and reproduction in any medium or format, as long as you give appropriate credit to the original author(s) and the source, provide a link to the Creative Commons license, and indicate if changes were made. The images or other third party material in this article are included in the article's Creative Commons license, unless indicated otherwise in a credit line to the material. If material is not included in the article's Creative Commons license and your intended use is not permitted by statutory regulation or exceeds the permitted use, you will need to obtain permission directly from the copyright holder. To view a copy of this license, visit http://creativecommons.org/licenses/by/4.0/.

(c) The Author(s) 2019 